\title{
Bifurcations of relative equilibria in the 4- and 5-body problem
}

\author{
KENNETH R. MEYER AND DIETER S. SCHMIDT \\ Department of Mathematical Sciences; Department of Computer Science, University \\ of Cincinnati, Cincinnati, Ohio 45221, USA
}

\begin{abstract}
The equilateral triangle family of relative equilibria of the 4-body problem consists of three particles of mass 1 at the vertices of an equilateral triangle and the fourth particle of arbitrary mass $m$ at the centroid. For one value of the mass $\boldsymbol{m}$ this relative equilibrium is degenerate. We show that families of isosceles triangle relative equilibria bifurcate from the equilateral triangle family as $m$ passes through the degenerate value.

The square family of relative equilibria of the 5-body problem consists of four particles of mass 1 at the vertices of a square and the fifth particle of arbitrary mass $m$ at the centroid. For one value of the mass $m$ this relative equilibrium is degenerate. We show that families of kite and isosceles trapezoidal relative equilibria bifurcate from the square family as $m$ passes through the degenerate value.
\end{abstract}

\section{Introduction}

A classical problem in celestial mechanics is to enumerate all the relative equilibria in the planar $N$-body problem. A relative equilibrium (RE) is an equilibrium solution of the equations of the $N$-body problem when written in uniformly rotating coordinates. For $N=3$ the collinear solutions of Euler [4] and the equilateral triangle solutions of Lagrange [7] complete the enumeration. For $N \geq 4$ the problem seems too difficult for a complete solution, so partial answers suffice. For all $N$ the collinear solutions are given in [8] or [10] and highly symmetric solutions are found in [2], [6], [9] and [12].

We will search for new special RE by using bifurcation analysis. Palmore [11] showed that there were one-parameter families of RE in the 4- and 5-body problem which became degenerate for one value of the parameter. This degeneracy suggests that a bifurcation occurs and that new RE are generated. We shall study the degeneracies of Palmore to illuminate the exact nature of the bifurcations.

For $N=4$ Palmore considered the one-parameter family consisting of three bodies of mass 1 at the vertices of an equilateral triangle and a fourth body of arbitrary mass $m$ at the centroid (figure 1(a)). We shall call this the equilateral RE (for the 4-body problem). He showed that $m_{4}^{*}=(81+64 \sqrt{3}) / 249$ is the unique value of the mass parameter $\boldsymbol{m}$ for which this RE is degenerate. We shall reproduce this result and prove that another family of RE bifurcate from the equilateral RE when $m=m_{4}^{*}$. The other family, called the isosceles RE, has three bodies of mass 1 at the vertices 


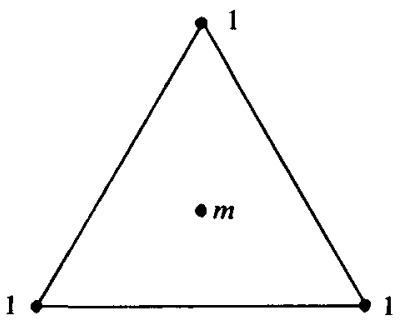

(a)

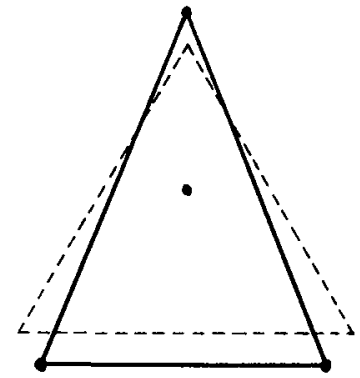

(b)

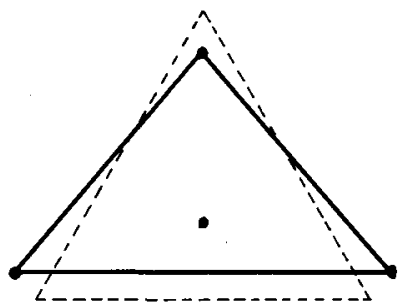

(c)

FIGURE 1. (a) Equilateral triangle. (b) Isosceles triangle for $m<m_{4}^{*}$. (c) Isosceles triangle for $m>m_{4}^{*}$.

of an isosceles triangle and a fourth body of mass $m$ near the centroid and on the line of symmetry of the triangle. For $m=m_{4}^{*}$ the isosceles family coincides with the equilateral family. Let the isosceles triangle have angles $\theta, \theta$ and $\phi$. For $m>m_{4}^{*}$, $\phi>60$ and for $m<m_{4}^{*}, \phi<60$ (see figures $1(\mathrm{~b})$ and $1(\mathrm{c})$ ). Of course, because of the symmetry of the problem, there are two other isosceles families obtained by rotating the first one by $120^{\circ}$ and $240^{\circ}$. We show that there are no non-symmetric RE which bifurcate from the equilateral RE when $m=m_{4}^{*}$. That is, there are no scalene RE near the equilateral RE. This refutes a conjecture in [11].

For $N=5$ Palmore considered a similar one-parameter family consisting of four bodies of mass 1 at the vertices of a square and a fifth body of arbitrary mass $m$ at the centroid (figure 2(a)). We call this the square RE (for the 5-body problem). He showed that $m_{5}^{*}=(13+11 \sqrt{2}) / 12$ is the unique value of the mass parameter $m$ for which the square RE is degenerate. We reproduce this result and prove that a family of kite RE as shown in figure 2(b) bifurcates when $m>m_{5}^{*}$ and a family of isosceles trapezoidal RE as shown figure 2 (c) bifurcates when $m<m_{5}^{*}$. Both these-families have a reflexive symmetry and other families can be obtained by rotation. Again there are no non-symmetric RE which bifurcate from the square family.

Our procedure is fairly standard for a bifurcation problem. The novelty is our use of the coordinates used in the now almost forgotten paper by Dziobek [3] and the algebraic processor MACSYMA to handle the more tedious calculations.

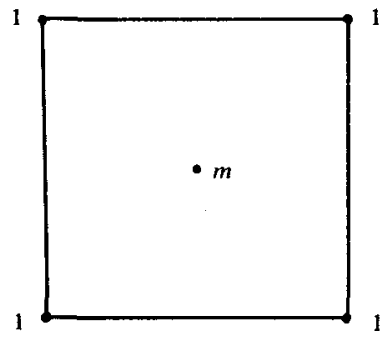

(a)

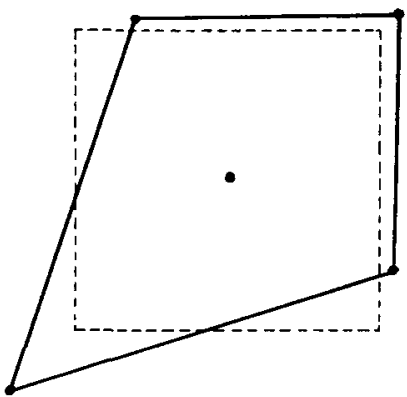

(b)

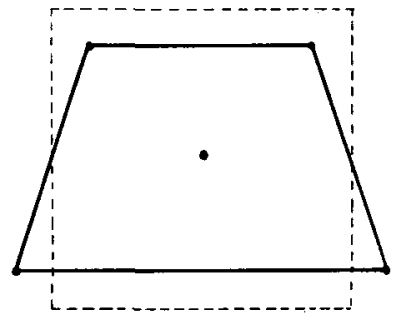

(c)

Figure 2. (a) Square family. (b) Kite for $m>m_{5}^{*}$. (c) Trapezoid for $m<m_{5}^{*}$. 


\section{Relative equilibria and the $N$-body problem}

The planar $\mathrm{N}$-body problem is defined by the system of ordinary differential equations

$$
m_{j} \ddot{q}_{j}=\partial U / \partial q_{j}, \quad j=1, \ldots, N,
$$

where $q_{j} \in \mathbb{R}^{2}$ is the position vector of the $j$ th body, $m_{j}>0$ is the mass of the $j$ th body and

$$
U=U(q)=\sum_{1 \leq i<j \leq N} m_{i} m_{j} /\left\|q_{j}-q_{i}\right\|
$$

is the (self) potential of the system. Thus the position of all $N$ bodies is specified by $q=\left(q_{1}, \ldots, q_{N}\right) \in \mathbb{R}^{2 N} \backslash \Delta$, where

$$
\Delta=\left\{q \in \mathbb{R}^{2 N}: \text { for some } i \text { and } j, i \neq j, q_{i}=q_{j}\right\} .
$$

For the moment think of $\mathbb{P}^{2}$ as $\mathbb{C}^{1}$ and the $q$ as complex numbers. To change to rotating coordinates, let $q_{j}=e^{i \omega t} u_{j}$, so equations (2.1) become

$$
m_{j}\left(\ddot{u}_{j}+2 i \omega \dot{u}_{j}-\omega^{2} u_{j}\right)=\partial U / \partial u_{j}, \quad j=1, \ldots, N
$$

and the condition for an equilibrium in these rotating coordinates is

$$
-\lambda u_{j}=\left(1 / m_{j}\right) \partial U / \partial u_{j}, \quad j=1, \ldots, N,
$$

where $\lambda=\omega^{2}$. Thus $u=\left(u_{1}, \ldots, u_{N}\right)$ is an RE if there is a $\lambda>0$ which satisfies the system of algebraic equations (2.4). By dotting with $u_{j}$, summing on $j$ and using Euler's theorem on homogeneous polynomials, one gets that $\lambda=U / 2 I>0$, where $I$ is the moment of inertia of the system, i.e.

$$
I=\frac{1}{2} \sum_{j=1}^{N} m_{j}\left\|u_{j}\right\|^{2} .
$$

Thus a solution of (2.4) forces a positive $\lambda$.

The variable $\lambda$ can be considered as a Lagrange multiplier and so an equivalent definition of an RE is a critical point of $U$ restricted to $S \backslash \Delta$, where $S=$ $\left\{u \in \mathbb{R}^{2 N}: I=I_{0}\right\}$, with $I_{0}>0$ a constant. If $u$ is an RE, then by multiplying (2.4) by $m_{j}$ and summing on $j$, one gets $\sum_{j=1}^{N} m_{j} u_{j}=0$ and so the centre of mass of the system is at the origin. If $u$ is an RE, then so is $\alpha u=\left(\alpha u_{1}, \ldots, \alpha u_{N}\right)$, where $\alpha=\rho e^{i \theta}$ is an arbitrary non-zero complex number. Thus any configuration of the bodies which is similar to an RE is also an RE. Thus RE lie in equivalence classes where the equivalence relation is similarity, i.e. $u \sim v$ if $\alpha u=v$, where $\alpha$ is a non-zero complex number. In the counting of RE one counts equivalence classes.

The concept of non-degenerate RE takes into account all the observations of the previous paragraph. Let $M=\left\{u \in \mathbb{R}^{2 N}: \sum m_{j} u_{j}=0\right\}, S$ as above and $\mathscr{S}=$ $\{(S \backslash \Delta) \cap M\} / \sim$, where $\sim$ is the equivalence relation defined above. Since $u \in S$ means $I(u)=I_{0}$, a constant, the scale of the system is fixed, so $u, v \in S$ and $u \sim v$ means $\alpha u=v$, where $\alpha=e^{i \theta}$ is a pure rotation. $U$ is invariant under rotations so constant on equivalence classes. Thus $\mathscr{U}: \mathscr{P} \rightarrow \mathbb{R}$ can be defined by $\mathscr{U}([u])=U(u)$. It turns out that $\mathscr{S}$ and $\mathcal{U}$ are smooth. An RE is called non-degenerate if its class is a non-degenerate critical point of $\mathscr{U}$ in the sense of Morse theory, i.e. the Hessian of $\mathcal{U}$ is non-singular at the critical point. 
One of the reasons that RE are difficult to find is that it is difficult to find good coordinates on $\mathscr{S}$. Here we will use the coordinates which were so artfully exploited in [3]. Dziobek's idea was to use the mutual distances $\rho_{i j}=\left\|u_{i}-u_{j}\right\|, 1 \leq i<j \leq N$, as coordinates, since these coordinates ignore the centre of mass and do not distinguish between configurations which are obtained from one another by a rotation. If the centre of mass is at the origin, then expanding $\rho_{i j}^{2}$ yields

$$
I=(1 / 4 M) \sum_{i=1}^{N} \sum_{j=1}^{N} m_{i} m_{j} \rho_{i j}^{2},
$$

where $M=\sum_{i=1}^{N} m_{i}$ is the total mass of the system. Thus $U$ and $I$ are functions of the mutual distances only. When $N=3$ the three positive numbers $\rho_{12}, \rho_{13}, \rho_{23}$ are coordinates for a non-collinear configuration provided they satisfy the inequalities imposed by the triangle inequality. In this case the dimension of $\mathscr{S}$ is 2 and so non-collinear classes in $\mathscr{S}$ have coordinates $\rho_{12}, \rho_{13}, \rho_{23}$ subject to the constraint $I=I_{0}$.

Thus to find the non-collinear RE for $N=3$, one needs to solve

$$
\left(\partial / \partial \rho_{i j}\right)\left\{U+\lambda\left(I-I_{0}\right)\right\}=-m_{i} m_{j} / \rho_{i j}^{2}+(\lambda / M) m_{i} m_{j} \rho_{i j}=0
$$

subject to the side condition $I=I_{0}\left(=M / 4\right.$ say). This gives $\rho_{i j}=1$ for all values of the masses. Moreover, this critical point is a non-degenerate minimum since the Hessian of $U+\lambda\left(I-I_{0}\right)$ is diagonal with positive diagonal entries. Thus for $N=3$ Dziobek's coordinates easily give that the only non-collinear RE are the nondegenerate equilateral triangle RE of Lagrange.

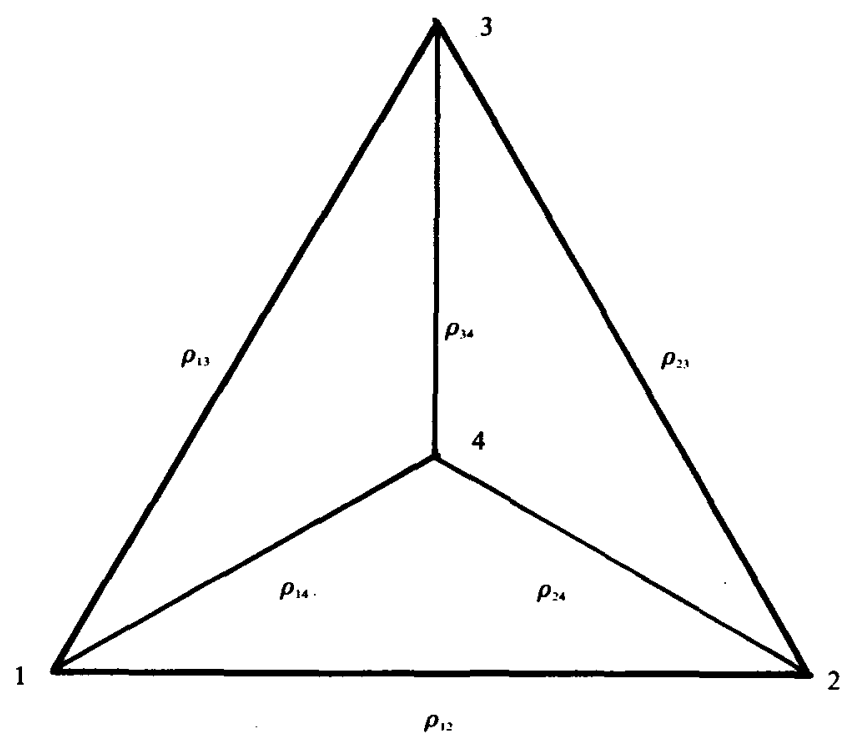

FIGURE 3. Mutual distances.

For $N=4$ there are six mutual distances (see figure 3 ). Clearly six numbers overdetermine the problem, and for four bodies in the general position five mutual distances suffice to determine the configuration. A necessary and sufficient condition 
that six positive numbers $\rho_{i j}, 1 \leq i<j \leq 4$, are the mutual distances between four coplanar points is

$$
F=\left|\begin{array}{ccccc}
0 & 1 & 1 & 1 & 1 \\
1 & 0 & r_{12} & r_{13} & r_{14} \\
1 & r_{12} & 0 & r_{23} & r_{24} \\
1 & r_{13} & r_{23} & 0 & r_{34} \\
1 & r_{14} & r_{24} & r_{34} & 0
\end{array}\right|=0,
$$

where $r_{i j}=\rho_{i j}^{2}$. The reader can verify that this 19 th century determinant is $288 \mathrm{~V}^{2}$, where $V$ is the volume of the tetrahedron whose six edges are given. This is the three-dimensional generalization of Heron of Alexandria's formula for the area of a triangle given its three sides.

Thus for $N=4$ we follow Dziobek and introduce a new Lagrange multiplier and look for critical points of $U+\lambda_{0}\left(I-I_{0}\right)+\left(\lambda_{1} / 2\right) F$ subject to the conditions $I=I_{0}$ and $F=0$. This is how we study the bifurcation problems in subsequent sections. A modern reference to this material is Hagihara [5].

\section{An illustrative example}

Before attacking the main problem, we shall look at a simple problem which models the essential features but lacks the technical difficulties of the full problem. This will indicate what to expect. Palmore [11] considers the equilateral triangle family for $N=4$ and the square family for $N=5$. In both cases the function $\mathcal{U}$ has a critical point for all values of $m$ and this critical point is degenerate for one specific value $m^{*}$ of $m$. He shows that at $m=m^{*}$ the nullity of the Hessian of $\mathcal{U}$ is 2 . In fact two of the eigenvalues of the Hessian change sign as $m$ passes through $m^{*}$.

Also from the symmetry it is clear that the function $u$ is invariant under the action of the appropriate dihedral group. Thus the essential features are (i) $\mathcal{U}$ is a one-parameter family of functions which has a fixed critical point for all values of the parameter, (ii) for one value of the parameter this critical point is degenerate with the Hessian having nullity 2 , (iii) the function is invariant under an action of the dihedral group. The perturbation parameter is $\varepsilon=m-m^{*}$.

Let $V_{\varepsilon}: \mathbb{R}^{2} \rightarrow \mathbb{R}$ be a smooth one-parameter family of functions which has the origin as a critical point for all values of $\varepsilon$. Since we are interested in critical points, we may assume $V_{\varepsilon}(0)=0$ also. Consider $\mathbb{R}^{2}$ as $\mathbb{C}^{1}$ and use $z$ and $\bar{z}$ as coordinates. Since $V_{\varepsilon}(0)=0$ and $\nabla V_{\varepsilon}(0)=0$, the Taylor expansion for $V_{\varepsilon}$ starts with quadratic terms. Assume $V_{\varepsilon}$ is invariant under the standard action of $D_{n}$ on $\mathbb{R}^{2}$, i.e. assume

$$
V_{\varepsilon}(z, \bar{z})=V_{\varepsilon}(\alpha z, \bar{\alpha} \bar{z}), \quad V_{\varepsilon}(z, \bar{z})=V_{\varepsilon}(\bar{z}, z),
$$

where $\alpha=\exp (2 \pi i / n)$. The only terms in a Taylor expansion which satisfy the first condition in (3.1) are of the form

$$
(z \bar{z})^{p} \bar{z}^{n q} \quad \text { or } \quad(z \bar{z})^{p} z^{n q},
$$

where $p$ and $q$ are non-negative integers. The second condition is simply a reality condition. Thus $V_{\varepsilon}$ would have a Taylor expansion of the form

$$
V_{\varepsilon}=\alpha_{1}(z \bar{z})+\alpha_{2}(z \bar{z})^{2}+\cdots+\left(\beta_{1} z^{n}+\bar{\beta}_{1} \bar{z}^{n}\right)+\cdots \text {. }
$$


If $\beta_{1} \neq 0$, then replacing $z$ by $\exp (-i \phi / n) z$, where $\phi=\arg \beta_{1}$, rotates coordinates so that $\beta_{1}$ is positive. Since $V$ is real, so are the $\alpha$. The Hessian of $V_{\varepsilon}$ will have nullity 2 when $\varepsilon=0$ if, for example, we take $\alpha_{1}=\varepsilon$ (or some multiple of $\varepsilon$ ). With $\alpha_{1}=\varepsilon$ the origin changes from a non-degenerate maximum when $\varepsilon<0$ to a nondegenerate minimum when $\varepsilon>0$.

We see that the $\beta$ term is of smaller degree than the $\alpha_{2}$ term when $n=3$ and the other way around when $n \geq 5$. For $n=4$ the two terms are of the same degree. In the typical or generic case $\alpha_{2} \neq 0$ and $\beta_{1} \neq 0$.

Example 1, $n=3$. Consider

$$
V_{\varepsilon}=\varepsilon z \bar{z}-\frac{1}{3}\left(z^{3}+\bar{z}^{3}\right) .
$$

Here we have a very simple one-parameter family of functions which has all the essential features discussed above. We omit the $\alpha_{2}$ term since it is of higher degree and makes no essential contribution. To find the critical points, proceed:

$$
\begin{aligned}
& \partial V_{\varepsilon} / \partial z=\varepsilon \bar{z}-z^{2}=0, \\
& \varepsilon z \bar{z}-z^{3}=0, \\
& \varepsilon r^{2}-r^{3} e^{i 3 \theta}=0, \quad z=r e^{i \theta}, \\
& e^{3 i \theta}= \pm 1, \quad r= \pm \varepsilon .
\end{aligned}
$$

Thus for $\varepsilon>0$ there are three critical points on the circle $r=\varepsilon$ at angles $\theta=0,2 \pi / 3$, $4 \pi / 3$ and for $\varepsilon<0$ there are three critical points on the circle $r=-\varepsilon>0$ at angles $\theta=\pi / 3, \pi / 3+2 \pi / 3, \pi / 3+4 \pi / 3$ (see figure $4(\mathrm{a})$ ).

Example 2, $n \geq 5$. Consider

$$
V_{\varepsilon}=\varepsilon z \bar{z}-\frac{1}{2}(z \bar{z})^{2}+(1 / n)\left(z^{n}+\bar{z}^{n}\right) .
$$

As before, proceed:

$$
\begin{aligned}
& \partial V_{\varepsilon} / \partial z=\varepsilon \bar{z}-(z \bar{z}) \bar{z}+z^{n-1}=0, \\
& \varepsilon r^{2}-r^{4}+r^{n} e^{i n \theta}=0, \quad z=r e^{i \theta}, \\
& \varepsilon-r^{2} \pm r^{n-2}=0, \quad e^{i n \theta}= \pm 1, \\
& r=\varepsilon^{1 / 2} \mp \frac{1}{2} \varepsilon^{(n-3) / 2}+\cdots \quad \text { for } \varepsilon>0,
\end{aligned}
$$

where $\theta=2 k \pi / n$ for $k=0, \ldots, n-1$ when the plus sign is taken and $\theta=$ $\pi / n+2 k \pi / n$ for $k=0, \ldots, n-1$ when the minus sign is taken (see figure $4(\mathrm{~b})$ ).

Example 3, $n=4$. Consider

$$
V_{\varepsilon}=\varepsilon(z \bar{z})-(\alpha / 2)(z \bar{z})^{2}+(\beta / 4)\left(z^{4}+\bar{z}^{4}\right)
$$

Proceed:

$$
\begin{aligned}
& \partial V_{\varepsilon} / \partial z=\varepsilon \bar{z}-\alpha(z \bar{z}) \bar{z}+\beta z^{3}=0, \\
& \vdots \\
& \varepsilon-(\alpha \mp \beta) r^{2}=0, \quad e^{i 4 \theta}= \pm 1, \\
& r^{2}=\varepsilon /(\alpha \mp \beta), \quad e^{i 4 \theta}= \pm 1 .
\end{aligned}
$$

There are two cases: $|\alpha|>|\beta|$ (case 1) and $|\alpha|<|\beta|$ (case 2). ( $|\alpha|=|\beta|$ is exceptional.) In the first case $\alpha \mp \beta$ are of the same sign, say positive. Then there are two groups of four critical points each for $\varepsilon>0$ and none for $\varepsilon<0$. In the second case $\alpha \mp \beta$ 

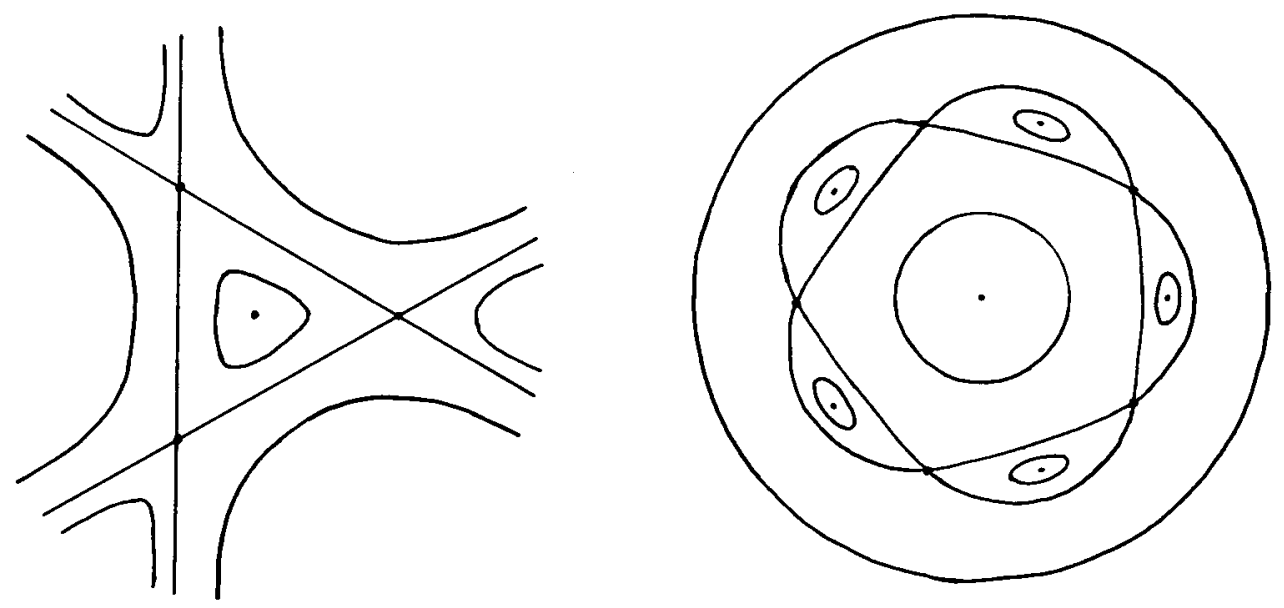

Figure 4. (a) Example 1, $\varepsilon>0$. (b) Example 2, $N=5, \varepsilon>0$.

have different signs and there is one group of four critical points for $\varepsilon>0$ and another group of four critical points when $\varepsilon<0$. The first case is like example 2 and the second case is like example 1.

In all the examples one can compute the second derivatives at the critical points just found and show that they are non-degenerate minima, maxima and saddles as illustrated in figures $4(a)$ and $4(b)$.

\section{The 4-body problem}

In this section we shall consider the bifurcations of the equilateral triangle family of the 4-body problem. Thus as indicated in $\$ 2$ we must find extrema of $U$ subject to the constraint $I=I_{0}$, and we shall use the mutual distances $\rho_{i j}$ as coordinates, which imposes the constraint $F=0$ (see (2.8)). Define

$$
V=U+\lambda_{0}\left(I-I_{0}\right)+\left(\lambda_{1} / 2\right) F,
$$

where these functions are defined as in $\S 2 . V$ is a function of eight variables which we order by introducing the 8-vector $z=\left(\lambda_{0}, \lambda_{1}, \rho_{12}, \rho_{13}, \rho_{14}, \rho_{23}, \rho_{24}, \rho_{34}\right)$. Henceforth the variables and equations will be numbered according to this convention. The equations to be solved are

$$
\partial V / \partial z=0
$$

or

$$
\begin{aligned}
& \partial V / \partial \lambda_{0}=I-I_{0}=0, \\
& \partial V / \partial \lambda_{1}=\frac{1}{2} F=0, \\
& \partial V / \partial \rho_{i j}=-m_{i} m_{j} / \rho_{i j}^{2}+\lambda_{0} m_{i} m_{j} \rho_{i j}+\lambda_{1}\left(\partial F / \partial r_{i j}\right) \rho_{i j}=0 .
\end{aligned}
$$

The last six equations are easier to handle if the equations are divided by $\rho_{i j}$ to give

$$
\left(1 / \rho_{i j}\right)\left(\partial V / \partial \rho_{i j}\right)=\left(\lambda_{0}-1 / \rho_{i j}^{3}\right) m_{i} m_{j}+\lambda\left(\partial F / \partial r_{i j}\right)=0 .
$$

Fix the masses by setting $m_{1}=m_{2}=m_{3}=1$ and $m_{4}=m$ arbitrary. Let the equations 
with (4.4) replacing the last six equations in (4.3) be denoted by $W=W(z, m)=0$. The equilateral triangle family $\rho_{12}=\rho_{13}=\rho_{23}=\sqrt{3}, \rho_{14}=\rho_{24}=\rho_{34}=1$ corresponds to the solution $z=a$, where

$$
a=((3 m+\sqrt{3}) /(3 m+9),(\sqrt{3}-9) m /(27 m+81), \sqrt{3}, \sqrt{3}, 1, \sqrt{3}, 1,1)
$$

and $I_{0}=(9+3 m) / 2$. Thus $W(a, m)=0$ for all $m$.

With the aid of MACSYMA the determinant of $\partial W / \partial z(a, m)$ is

$$
\{(532 \sqrt{3}-720) / 6889\} m^{2}\{249 m-64 \sqrt{3}-81\}^{2},
$$

which is non-zero for all positive $m$ except $m=m_{4}^{*}=(64 \sqrt{3}+81) / 249$. Thus the equilateral triangle family is non-degenerate for all $m \neq m_{4}^{*}$, which agrees with Palmore [11].

When $m=m_{4}^{*}$ the $6 \times 6$ submatrix obtained from $\partial W / \partial z$ by deleting the last two rows and columns has non-zero determinant. Thus by the implicit function theorem the first six equations can be solved to give $\lambda_{0}, \lambda_{1}, \rho_{12}, \rho_{13}, \rho_{14}, \rho_{23}$ in terms of $\rho_{24}$, $\rho_{34}$ and $m$ for $m$ near $m_{4}^{*}$. This affects the Liapunov-Schmidt reduction.

Let $m=m_{4}^{*}+\varepsilon, \quad z=a+\varepsilon b+\cdots, Z=\left(W_{7}, W_{8}\right), \quad u=\left(z_{7}, z_{8}\right)=\left(\rho_{24}, \rho_{34}\right), \quad \alpha=$ $\left(a_{7}, a_{8}\right), \beta=\left(b_{7}, b_{8}\right)$. Solving the first six equations order by order and substituting into the last equations yields

$$
Z(u, \varepsilon)=0,
$$

which is identically zero at order 0 and 1. At order 2 equations (4.7) become

$$
\left(2 b_{7}+b_{8}\right)\left(p_{1} b_{8}+p_{2}\right)=0, \quad\left(b_{7}+2 b_{8}\right)\left(p_{1} b_{7}+p_{2}\right)=0,
$$

where $p_{1}$ and $p_{2}$ are non-zero numeric constants given in the Appendix. Besides the trivial solution $b_{7}=b_{8}=0$, there are the following three pairs of solutions:

$$
\begin{array}{ll}
b_{7}=b_{8}=-p_{3}, \\
b_{7}=-p_{3}, \quad b_{8}=2 p_{3}, \\
b_{7}=2 p_{3}, \quad b_{8}=-p_{3},
\end{array}
$$

where $p_{3}=p_{1} / p_{2}$. The fact that there are three non-trivial solutions is a consequence of the threefold symmetry of the problem, so concentrate on the first. The Jacobian of equations (4.8) at the solution (4.9) is $-p_{1}^{2} \neq 0$, so the implicit function theorem says that equations (4.7) can be solved for $u$ as a function of $\varepsilon$ with $u=$ $-\left(p_{3}, p_{3}\right)+O(\varepsilon)$. This means the last two distances $\rho_{24}$ and $\rho_{34}$ can be expanded as series in $\varepsilon$ and $\rho_{24}=\rho_{34}=1-\varepsilon p_{3}+O\left(\varepsilon^{2}\right)$. Previously, we had found that the first six equations of (4.2) or (4.3) can be solved for the first six variables as a function of the last two, $\rho_{24}$ and $\rho_{34}$, and $m$. Combining these results gives a series solution $z=a+\varepsilon b+\cdots$ of equations (4.2) and (4.3), where $a$ and $b$ are given in the Appendix. The last two components of $b$ are as found above to be $-p_{3} \neq 0$. The remaining components were obtained by substituting these values into the previously computed series for the first six variables.

Thus we have shown that the equilateral triangle family of relative equilibria of the 4-body problem experiences a bifurcation at $m_{4}^{*}$. From the symmetry of the equations it is easy to see that for the solution found $\rho_{24} \equiv \rho_{34}$ and $\rho_{12} \equiv \rho_{13}$, i.e. the solution has a $Z_{2}$ symmetry and the configuration has three bodies at the vertices 
of an isosceles triangle and one in the interior (see figures $1(\mathrm{~b})$ and $1(\mathrm{c})$ ). Moreover, from the symmetry of the equations the three solutions of (4.8) give rise to three series expansions for $z$ as a function of $\varepsilon=m-m_{4}^{*}$, and these three solutions are obtained from one another by a rotation of the configuration by $2 \pi / 3$.

If existence were our only goal, we could have simplified the problem by seeking only symmetric solutions. We did not do this, but decided to treat the full set of equations in case non-symmetric solutions made a bifurcation also. Our analysis shows that the only solutions which bifurcate from the equilateral family are the three isosceles families discussed above. This follows from the uniqueness of solutions when the implicit function theorem is applied. This disproves a conjecture of Palmore [11] on the bifurcation of scalene families.

The essential features found in the relative equilibrium problem are the same as in the illustrated example in $\$ 3$. In the case of a threefold symmetry there is a bifurcation of three solutions which respect the $Z_{2}$ symmetry of the problem. Also the bifurcated solutions are expandable in the first power of the perturbation parameter and therefore exist when the parameter is on both sides of the critical value.

\section{The 5-body problem}

In this section we shall consider the bifurcation of the square family of relative equilibria of the 5-body problem by the same method as used in the previous section for the 4-body problem. Since only minor modifications need to be made, only an outline will be presented. In the 5-body problem there are 10 mutual distances $\rho_{i j}$, $1 \leq i<j \leq 5$, so three constraints are necessary to insure that these 10 numbers represent the mutual distances between five points in the plane. Let $F(i, j, k, l)$ be the determinant given in $(2.8)$ with $(i, j, k, l)$ replacing $(1,2,3,4)$ in that order. Then the three constraints we choose are $F_{1}=F(1,2,3,4)=0, F_{2}=F(1,2,3,5)=0$ and $F_{3}=F(1,2,4,5)=0$.

Consider

$$
V=U+\lambda_{0}\left(I-I_{0}\right)+\left(\lambda_{1} / 2\right) F_{1}+\left(\lambda_{2} / 2\right) F_{2}+\left(\lambda_{3} / 2\right) F_{3},
$$

so $V$ is a function of 14 variables which are ordered by the vector

$$
z=\left(\lambda_{0}, \lambda_{1}, \lambda_{2}, \lambda_{3}, \rho_{12}, \rho_{13}, \rho_{14}, \rho_{15}, \rho_{23}, \rho_{24}, \rho_{25}, \rho_{34}, \rho_{35}, \rho_{45}\right) \text {. }
$$

The equations to be solved are

$$
\partial V / \partial z=0
$$

and again we replace $\partial V / \partial \rho_{i j}=0$ by $\left(1 / \rho_{i j}\right)\left(\partial V / \partial \rho_{i j}\right)$ in (5.3) to obtain the equivalent system

$$
W(z, m)=0 .
$$

The square family chosen is $\rho_{15}=\rho_{25}=\rho_{35}=\rho_{45}=1, \rho_{12}=\rho_{14}=\rho_{23}=\rho_{34}=\sqrt{2}, \rho_{13}=$ $\rho_{24}=2, m_{1}=m_{2}=m_{3}=m_{4}=1$ and $m_{5}=m$ arbitrary. The corresponding values of the $\lambda$ are computed to give a vector $a$ such that

$$
W(a, m)=0 \text {. }
$$

MACSYMA computes $\operatorname{det}(\partial W / \partial z)(a, m)$ to be

$$
q_{0} m^{2}(4 m+1)\{12 m-13-11 \sqrt{2}\}^{2}
$$


where $q_{0}$ is a negative constant. This determinant is non-zero for positive $m$ except $m=m_{5}^{*}=(13+11 \sqrt{2}) / 12$. Thus the square family of relative equilibria is nondegenerate except when $m=m_{5}^{*}$, which agrees with Palmore [11]. The $12 \times 12$ submatrix of $\partial W / \partial z$ obtained by deleting the last two rows and columns is nonsingular, and so the first 12 equations can be solved for the first 12 variables and the result substituted into the last two equations. This affects the Liapunov-Schmidt reduction. Let $z=a+\varepsilon b+\varepsilon^{2} c+\cdots$, where $m=m_{5}^{*}+\delta \varepsilon^{2}, \delta= \pm 1, Z=\left(W_{13}, W_{14}\right)$, $U=\left(z_{13}, z_{14}\right)=\left(\rho_{35}, \rho_{45}\right), \alpha=\left(a_{13}, a_{14}\right), \beta=\left(b_{13}, b_{14}\right), \gamma=\left(c_{13}, c_{14}\right)$. The problem is reduced to solving

$$
Z(u, \varepsilon)=0,
$$

which vanishes identically in $\varepsilon$ up to order 2 . The first non-zero terms occur at order $\varepsilon^{3}$ and are of the form

$$
\begin{aligned}
& \left(q_{1} \delta-q_{2} b_{13}^{2}+q_{3} b_{14}^{2}\right)\left(b_{13} / q_{4}\right)=0, \\
& \left(q_{1} \delta+q_{3} b_{13}^{2}-q_{2} b_{14}^{2}\right)\left(b_{14} / q_{4}\right)=0,
\end{aligned}
$$

where $q_{1}, q_{2}, q_{3}, q_{4}$ are positive constants which are listed in the Appendix. Since we seek real solutions, we first set $\delta=+1$ then $\delta=-1$. When $\delta=+1$, besides the trivial solution there are the solution pairs

$$
\begin{aligned}
& b_{13}=0, \quad b_{14}= \pm q_{1} / q_{2}, \\
& b_{13}= \pm q_{1} / q_{2}, \quad b_{14}=0,
\end{aligned}
$$

and when $\delta=-1$ the non-trivial solutions are

$$
b_{13}= \pm q_{5}, \quad b_{14}= \pm q_{5},
$$

where $q_{5}=\sqrt{q_{1}\left(q_{3}-q_{2}\right) /\left(q_{2}^{2}+q_{3}^{2}\right)}$. The Jacobians of equations $(5.8)$ are seen to be non-singular at the solutions (5.9) and (5.10), so the implicit function theorem yields a solution of (5.7) and hence of (5.5) for each of the solutions in (5.9) and (5.10). There are eight such solutions in two groups of four, the groups resulting from the symmetry of the problem. When $\delta=+1, m>m_{5}^{*}$ and the family which bifurcates from the square family is symmetric with respect to one of its diagonals and gives rise to the kite family of relative equilibria as illustrated in figure 2(b). When $\delta=-1$, $m<m_{5}^{*}$ and the family which bifurcates from the square is a family of isosceles trapezoids as illustrated in figure $2(\mathrm{c})$.

As in the previous case, there are no non-symmetric solutions which bifurcate. This bifurcation is of the same general type as discussed in $\S 2$, example 3 , case 2 .

Acknowledgements. The authors would like to thank Dr Marcelo Crespo da Silva for help in using MACSYMA on a Symbolics machine. This research was supported by grants from NSF and ACMP/DARPA.

Appendix. Constants

$p_{1}=(521486264 \sqrt{3}+859264848) / 29732924$,

$p_{2}=-(15438249 \sqrt{3}+49745469) / 29732924$,

$a_{1}=(9 \sqrt{3}+3) / 52$,

$a_{2}=-(29 \sqrt{3}+27) / 1404$, 


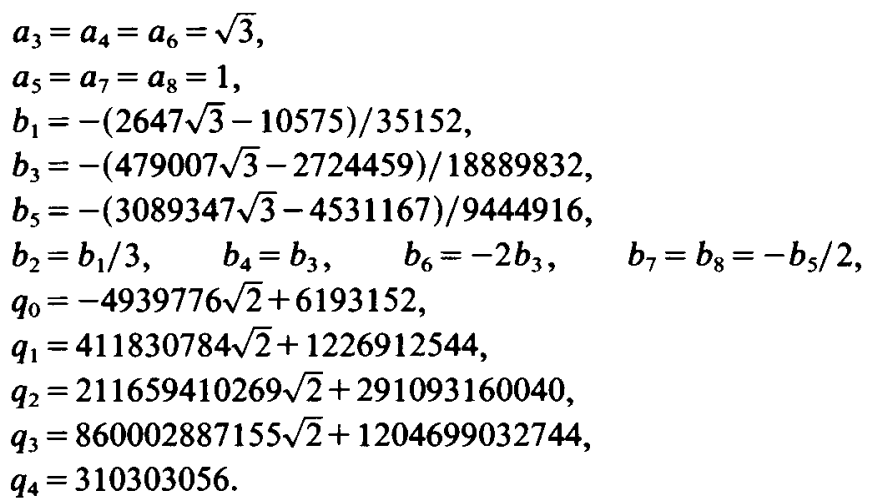

\section{REFERENCES}

[1] M. H. Andoyer. Sur l'équilibre relatif de $n$ corps. Bull. Astronomique 23 (1906), 50-59.

[2] E. Breglia. Su alcuni casi particolari del problema di tre corpi. Giorn. Math. 7 (1916), 3, $151-173$.

[3] O. Dziobek. Über einen merkwürdigen Fall des Vielkörperproblem. Astron. Nach. 152 (1900), 32-46.

[4] L. Euler. De motu restilineo trium corporum se mutus attrahentium. Novi Comm. Acad. Sci. Imp. Petrop. 11 (1767), 144-151.

[5] Y. Hagihara. Celestial Mechanics I. MIT Press, Cambridge, MA (1970).

[6] R. Hoppe. Erweiterung der bekannten Speciallösung des Dreikörper problems. Archiv. Math. Phys. 64 (1879), 218-223.

[7] J. L. Lagrange. Essai sur le problem des trois corps. Oeuvres 6 (1772), 272-292.

[8] R. Lehmann-Filhes. Über zwei Fälle des Vielkörpersproblem. Astron. Nach. 127 (1891), 137-144.

[9] W. R. Longley. Some particular solutions in the problem of $n$-bodies. Bull. Amer. Math. Soc. 13 (1906), 324-335.

[10] F. R. Moulton. The straight line solutions of the problem of N-bodies. Ann. Math. 12 (1910), 2, 1-17.

[11] J. I. Palmore. Relative equilibria of the $n$-body problem. Thesis. University of California, Berkeley, CA (1973).

[12] A. Wintner. The Analytic Foundations of Celestial Mechanics. Princeton Mathematics Series 5. Princeton University Press, Princeton, NJ (1941). 